ACCEPted By The Astrophysical Journal

Preprint typeset using $\mathrm{LAT}_{\mathrm{E}} \mathrm{X}$ style emulateapj v. 8/13/10

\title{
NUSTAR DETECTION OF QUIESCENT HARD X-RAY EMISSION FROM SGR 0526-66 IN THE LARGE MAGELLANIC CLOUD
}

\author{
Sangwook Park ${ }^{1,4}$, Jayant Bhalerao ${ }^{1}$, Oleg Kargaltsev ${ }^{2}$, and Patrick O. Slane ${ }^{3}$ \\ Accepted by The Astrophysical Journal
}

\begin{abstract}
The soft $\gamma$-ray repeater (SGR) 0526-66 is the first-identified magnetar, and is projected within the supernova remnant N49 in the Large Magellanic Cloud. Based on our 50 ks NuSTAR observation, we detect the quiescent-state $0526-66$ for the first time in the $10-40 \mathrm{keV}$ band. Based on the joint analysis of our NuSTAR and the archival Chandra ACIS data, we firmly establish the presence of the nonthermal component in the X-ray spectrum of 0526-66 in addition to the thermal emission. In the best-fit blackbody (BB) plus power law (PL) model, the slope of the PL component (photon index $\Gamma$ $=2.1)$ is steeper than those $(\Gamma \lesssim 1.5)$ for other magnetars. The soft part of the X-ray spectrum can be described with a $\mathrm{BB}$ component with the temperature of $k T=0.43 \mathrm{keV}$. The best-fit radius $(R=$ $6.5 \mathrm{~km}$ ) of the X-ray-emitting area is smaller than the canonical size of a neutron star. If we assume an underlying cool $\mathrm{BB}$ component with the canonical radius of $R=10 \mathrm{~km}$ for the neutron star in addition to the hot $\mathrm{BB}$ component (2BB $+\mathrm{PL}$ model), a lower $\mathrm{BB}$ temperature of $k T=0.24 \mathrm{keV}$ is obtained for the passively cooling neutron star's surface, while the hot spot emission with $k T=0.46$ $\mathrm{keV}$ dominates the thermal spectrum $(\sim 85 \%$ of the thermal luminosity in the $0.5-5 \mathrm{keV}$ band $)$. The nonthermal component $(\Gamma \sim 1.8)$ is still required.
\end{abstract}

Subject headings: X-rays: individual (SGR 0526-66) — stars: neutron — X-rays: stars

\section{INTRODUCTION}

The soft $\gamma$-ray repeater (SGR) 0526-66 showed intense $\gamma$-ray outbursts on 1979 March 5 and a luminous pulsed afterglow with $P \sim 8$ s (Mazets et al. 1979; Cline et al. 1980), which led to the discovery of the magnetar phenomenon. The quiescent soft X-ray counterpart is projected within the supernova remnant (SNR) N49 in the Large Magellanic Cloud (LMC) (Rothschild et al. 1994), and its pulsations with $P=$ $8.0436 \mathrm{~s}$ and $\dot{P}=6.6 \times 10^{-11} \mathrm{~s} \mathrm{~s}^{-1}$ were detected with a low pulsed-fraction of $f_{p} \sim 10 \%$ in the Chandra data (Kulkarni et al. 2003). Later Chandra and XMMNewton data showed that the spin-down rate $(\dot{P})$ might have decreased by $\sim 40 \%$ over the course of several years (Tiengo et al. 2009; Güver et al. 2012). The estimated pulsed-fraction appeared to decrease to $f_{p} \sim 4 \%$ by 2009 (Güver et al. 2012). Evidence of a slow decay ( 20-30\% in $\sim 17$ yr until 2009) in the soft X-ray flux was reported, which may be related to its long-term surface cooling and/or evolution of the X-ray emitting hot spot areas (Park et al. 2012; Güver et al. 2012).

The $1-10 \mathrm{keV}$ band XMM-Newton spectrum can be fitted with a simple power law (PL) model with a steep photon index $(\Gamma \sim 3.3)$ (Tiengo et al. 2009). The $0.5-$ $6 \mathrm{keV}$ band Chandra spectrum (based on simultaneous spectral model fits of six data sets taken in 2000, 2001, and 2009 with $\sim 30-50 \mathrm{ks}$ individual exposures) was fitted with spectral models for the X-ray emission from a magnetized H-atmosphere $(k T \sim 0.35 \mathrm{keV})$ of a neu-

\footnotetext{
${ }^{1}$ Box 19059, Department of Physics, University of Texas at Arlington, Arlington, TX 76019

2 Department of Physics, George Washington University, 725

21st Street NW, Washington, DC 20052

${ }^{3}$ Harvard-Smithsonian Center for Astrophysics, 60 Garden Street, Cambridge, MA 02138

${ }^{4}$ s.park@uta.edu
}

tron star (Güver et al. 2012). The Chandra data with higher photon count statistics (with $\sim 110 \mathrm{ks}$ effective exposure in total, combining four observations taken in 2009) showed that two-component models, e.g., thermal blackbody (BB) and/or nonthermal PL components, are required to adequately fit the observed X-ray spectrum in the $0.4-8 \mathrm{keV}$ band (Park et al. 2012). In the twocomponent spectral model fits, the soft component of the observed X-ray spectrum might be attributed to emission from the surface cooling neutron star (a BB-like emission with $k T \sim 0.4 \mathrm{keV})$. Depending on the adopted spectral models, a hot spot(s) (e.g., BB with $k T \sim 1 \mathrm{keV}$ and $R \sim$ $1 \mathrm{~km}$ ) or the nonthermal magnetospheric radiation (e.g., a PL with $\Gamma \sim 2.5$ ) were required to fit the remaining part of the X-ray spectrum, primarily in the $\sim 3-8 \mathrm{keV}$ band.

Broadband X-ray spectroscopy covering $E>10 \mathrm{keV}$ is crucial to establish the properties of the nonthermal component and to discriminate between the hotter thermal and nonthermal emission. Here we report the results from our $\sim 50$ ks $N u S T A R$ observation, which provides the detection of the hard X-ray emission from 0526-66 up to $E \sim 40 \mathrm{keV}$. We also present results from our joint analysis of the broadband X-ray spectrum (in the 0.4 - $40 \mathrm{keV}$ band) of $0526-66$ based on our NuSTAR and the archival Chandra data. We describe the observations and the data reduction in Section 2. We present our data analysis in Sections $3 \& 4$, and a discussion in Section 5 . A summary is presented in Section 6 .

\section{OBSERVATIONS \& DATA}

We observed $0526-66$ with $N u S T A R$ on 2018 February 7 during Cycle 3. We processed the data with NuSTARDAS (NuSTAR Data Analysis Software) version 1.7.1 and NuSTAR CALDB version 20180126. After the standard data reduction, the effective exposure is $47 \mathrm{ks}$. We 
extracted the NuSTAR spectrum of $0526-66$ from a circular source region with a radius of $30^{\prime \prime}$, centered on the source position RA (J2000) $=05^{h} 26^{m} 01^{s} .61$, and Dec $(\mathrm{J} 2000)=-66^{\circ} 0446 !^{\prime \prime} 0$. We extracted the background spectrum from an annular region around the source. The source and background spectra and spectral response files were created with the standard HEASARC software NUPIPELINE tool. Combining the data taken with two Focal Plane Modules (FPMA and FPMB), we extracted $\sim 1200$ counts from the source extraction region in the 2 $-40 \mathrm{keV}$ band. We estimate that $\sim 25 \%$ of them are due to the background, while the remaining ( $\sim 900$ counts) comprises both the SGR and emission from SNR N49 (Figure 1 and Section 3). Based on our spectral model fits (see Section 3), we estimate the total background including the contamination by $\mathrm{N} 49$ is $\sim 60 \%$ of the total extracted counts in the $2-40 \mathrm{keV}$ band. In the $10-40$ $\mathrm{keV}$ band, we extract $\sim 280$ counts, $\sim 50 \%$ of which is the background. The contamination from SNR N49 is negligible in this band. Thus, we make a clear $\sim 8 \sigma(\sim 140$ background-subtracted counts) detection of $0526-66$ in the $10-40 \mathrm{keV}$ band.

About $1 / 4$ of the field of view in the north-northeast of the source was moderately affected by scattered X-rays from nearby bright X-ray sources (Figure 1). The lowmass X-ray binary LMC X-3, which is projected at $\sim 2^{\circ} .4$ northeast of $0526-66$, is the only bright $\mathrm{X}$-ray source within a few degrees in the north-northeast of 0526-66. Thus, although LMC X-3 was not in a high state during our NuSTAR observation of $0526-66$ (based on the archival MAXI data), the contaminating source appears to be LMC X-3. 0526-66 is positioned close to (or just outside of) the boundary of the affected background region (Figure 11). Based on our background spectral extractions from several regions around the source, we estimated a $\sim 10 \%$ contamination from these scattered $\mathrm{X}$ rays on the $10-40 \mathrm{keV}$ band source flux, which would not significantly affect our analysis. In the following sections we assume the average background spectrum, extracted from an annular region around the source (Figure 1).

As supplementary data to model the broadband X-ray spectrum of $0526-66$, we jointly analyzed the archival Chandra data of 0526-66 taken with the Advanced CCD Imaging Spectrometer (ACIS) in 2009 (ObsIDs 10123, 10806,10807 , and 10808) $\sqrt{5}$. A $1 / 4$ subarray of the ACISS3 detector was used in these archival Chandra data, which ensured a low photon pileup $(<5 \%)$ for a reliable spectral analysis of 0526-66. We processed each of the raw data sets and merged them, following the standard data reduction methods as described in Park et al. (2012). We extracted the source spectrum from a circular region centered on the source position $\left(2^{\prime \prime}\right.$ in radius). Since $0526-66$ is projected within the boundary of

\footnotetext{
5 This is a "single" observation, which was split into four subsequences over a relatively short two-month period to accommodate the restrictions on the solar pitch angles for the telescope. There are several other Chandra archival data sets of 0526-66, which were taken several years earlier with shorter exposures than these 2009 data. Taking advantage of the longest effective exposure and the closest epoch of the observation (to that of our NuSTAR observation), we use these 2009 Chandra data for the joint spectral analysis in this work. The large PSF of XMM-Newton resulted in a significant contamination from SNR N49 in the soft X-ray band spectrum of 0526-66 (Tiengo et al. 2009). Thus, we do not use the archival $X M M$-Newton data in this work.
}

the X-ray emitting shell of SNR N49, the background characterization based on the high resolution imaging spectroscopy with Chandra ACIS data is critical. We extracted the background spectrum from an annular region around the source within SNR N49. Based on these data we extract $\sim 21000$ photons (including $\sim 12 \%$ background) for $0526-66$ in the $0.4-8 \mathrm{keV}$ band.

\section{X-RAY SPECTRAL ANALYSIS}

The main goal of the NUSTAR observation of $0526-$ 66 is to search for hard X-ray emission at $E \gtrsim 10 \mathrm{keV}$. While we make a clear detection of $0526-66$ in the 10 - $40 \mathrm{keV}$ band, the utility of the NuSTAR data alone to adequately characterize the soft X-ray spectrum of 0526-66 is limited, because of the poor count statistics in the soft X-ray band (i.e., no response at $E<$ $2 \mathrm{keV}$ for FPM and the significant contamination from SNR N49's thermal emission at $E<5 \mathrm{keV}$ ). Thus, we perform the NuSTAR + Chandra joint spectral analysis to fit the broadband X-ray spectrum of $0526-66$. For our spectral analysis we rebinned both of NuSTAR and Chandra spectra to contain a minimum of 20 counts per energy channel, and perform all spectral model fits in the $0.4-40 \mathrm{keV}$ band. In our spectral model fits, we fixed the Galactic column at $N_{\text {H.Gal }}=6 \times 10^{20} \mathrm{~cm}^{-2}$ toward 0526-66 (Dickey \& Lockman 1990). We fit the foreground column in the LMC $\left(N_{\mathrm{H}, \mathrm{LMC}}\right)$ assuming the LMC abundances available in the literature $(\mathrm{He}=0.89$, $\mathrm{C}=0.30, \mathrm{~N}=0.12, \mathrm{O}=0.26, \mathrm{Ne}=0.33, \mathrm{Na}=0.30, \mathrm{Mg}$ $=0.32, \mathrm{Al}=0.30, \mathrm{Si}=0.30, \mathrm{~S}=0.31, \mathrm{Cl}=0.31, \mathrm{Ar}=$ $0.54, \mathrm{Ca}=0.34, \mathrm{Cr}=0.61, \mathrm{Fe}=0.36, \mathrm{Co}=0.30$, and $\mathrm{Ni}=0.62$, Russell \& Dopita 1992; Hughes et al. 1998). Hereafter, elemental abundances are with respect to solar (Anders \& Grevesse 1989). We tested recent X-ray measurements of the LMC abundances for $\mathrm{O}, \mathrm{Ne}, \mathrm{Mg}$, and $\mathrm{Fe}$, which were based on the X-ray spectral model fits of the shocked interstellar medium in the LMC SNRs (Maggi et al. 2016; Schenck et al. 2016) for our $N_{\text {H.LMC }}$ parameter. These LMC abundance measurements are lower than the previous values by $\sim 30-50 \%$, which resulted in slight increases (by $\sim 10-20 \%$ ) for the best-fit $N_{\mathrm{H}, \mathrm{LMC}}$. Otherwise, the impact of these LMC abundances on the results from our spectral model fits of 0526-66 is not statistically significant, and thus does not affect our conclusions. For self-consistent comparisons with the previous results in the literature, we assumed the LMC abundances listed above in this work.

In our spectral model fits, we tied $N_{\mathrm{H}, \mathrm{LMC}}$, the $\mathrm{BB}$ temperature, the BB-emitting area, the PL photon index and normalization (see Sections 3.1 \& 3.2 for our adopted spectral models) between the NUSTAR and Chandra spectra. At $E \lesssim 7 \mathrm{keV}$, the contamination from the soft thermal X-ray emission from SNR N49 (whose angular extent is $\sim 1^{\prime}$, Figure (1) is substantial in the NuSTAR spectrum of $0526-66$ due to the large PSF (58" HPD, Harrison et al. 2013) of NuSTAR. To account for this SNR spectrum, we added a plane-shock (PS) component (with the electron temperature of the hot gas $k T$ $\sim 1 \mathrm{keV}$ on average, based on Park et al. [2003] and Uchida et al. [2015]) in the spectral modeling of the NuSTAR spectrum of $0526-66$. We find that this background emission component from SNR N49 contributes $\sim 40 \%$ of the NuSTAR flux of $0526-66$ in the $2-7 \mathrm{keV}$ band, and is negligible ( $<5 \%$ of the total flux) in the 7 
- $40 \mathrm{keV}$ band. Thanks to the sub-arcsecond resolution of the Chandra ACIS data, the contamination from the SNR in the Chandra spectrum of $0526-66$ is small. We subtracted it from the total spectrum of the source (Section 2), rather than applying this additional PS model component for the Chandra spectrum.

\subsection{Thermal Spectral Models for Hard Component}

There are several publicly-available spectral models for the X-ray spectrum emitted by the neutron star's hot atmosphere with various atomic compositions, such as the NSA model (Pavlov et al. 1995) and NSMAX models (Ho et al. 2008; Mori \& Ho 2007). While these atmospheric models are more physically-motivated to describe the observed X-ray spectra from neutron stars, they are not adequate for magnetars, whose magnetic fields are very strong: e.g., these models allow the magnetic fields up to $B \sim 10^{13} \mathrm{G}$, whereas $B \sim 10^{14-15}$ has been estimated for $0526-66$. Thus, we chose to use simple BB models for the thermal component of the X-ray spectrum of $0526-66$ in this work, realizing that they may provide only a phenomenological (rather than physically accurate) description of the data. We note that fits with the BB model still allow us to investigate the thermal vs. nonthermal nature of the X-ray spectrum, and would also enable self-consistent comparisons with the results from the magnetar analysis in the literature (where the BB models are commonly adopted).

In agreement with the previously published spectral analysis of the same Chandra observations (Park et al. 2012), we find that the single BB model cannot fit the observed broadband X-ray spectrum of 0526 $66\left(\chi^{2} / \nu \sim 3\right)$. Then, we applied two component spectral models. To test the thermal origin of the hard component of its X-ray spectrum, we fitted the broadband X-ray spectrum of 0526-66 with a two-component BB model. In this spectral model fit, we initially fixed the cool and hot component $\mathrm{BB}$ temperatures at the values derived by Park et al. (2012): i.e., $k T_{\text {soft }} \approx 0.4 \mathrm{keV}$ and $k T_{\text {hard }} \approx 1$ $\mathrm{keV}$. The best-fit model is statistically poor $\left(\chi^{2} / \nu \sim 1.5\right)$ because our $N u S T A R$ spectrum at $E \gtrsim 7 \mathrm{keV}$ cannot be fitted with the BB model. When it is fitted, the temperature of the hot $\mathrm{BB}$ component increases to $k T \approx 2 \mathrm{keV}$ for the improved best-fit $\left(\chi^{2} / \nu \sim 1.3\right)$. However, the systematic excess in the NuSTAR spectrum is still evident at $E \gtrsim 10 \mathrm{keV}\left(\chi^{2} / \nu=2.7\right.$ in the $10-40 \mathrm{keV}$ band $)$, even with this high temperature (Figure 2a). Thus, we conclude that the two-temperature BB model cannot adequately describe the $N u S T A R+$ Chandra spectrum of 0526-66.

\subsection{Nonthermal Spectral Models for Hard Component}

We first attempted a single PL model fit to the broadband X-ray spectrum of 0526-66. The best-fit PL model is statistically unacceptable $\left(\chi^{2} / \nu=1.8\right)$, showing significant residuals both in the soft $(E<2 \mathrm{keV})$ and the hard bands $(E>5 \mathrm{keV})$. Next, we fitted the broadband $\mathrm{X}$-ray spectrum of 0526 with $\mathrm{BB}+\mathrm{PL}$ models. The best-fit model results in $k T_{B B}=0.43 \mathrm{keV}$ and the PL photon index $\Gamma=2.10\left(\chi^{2} / \nu=1.09\right.$, Figures [2b \& 3] $)$. This best-fit $\mathrm{BB}+\mathrm{PL}$ model indicates an emission radius of the neutron star $R_{\mathrm{NS}} \approx 6.5 \mathrm{~km}$. These results are summarized in Table 1 .
Recent NuSTAR observations of magnetars and anomalous X-ray pulsars have shown the presence of similar hard X-ray emission components at $E>10$ $\mathrm{keV}$, for which the broadband X-ray spectrum was fitted with three-component $(2 \mathrm{BB}+\mathrm{PL})$ spectral models (e.g., Gotthelf et al. 2019). Taking a similar approach, we apply $2 \mathrm{BB}+\mathrm{PL}$ model fits for 0526-66. In these model fits, we assume the canonical radius of neutron stars $\left(R_{\mathrm{NS}}=10 \mathrm{~km}\right)$ for the emitting area of the soft component $\mathrm{BB}$ spectrum. The best-fit model $\left(\chi^{2} / \nu=\right.$ 1.09) implies $k T_{\mathrm{TH} 1}=0.24 \mathrm{keV}$ for the cooling surface of the neutron star. The best-fit hot spot temperature is $k T_{\mathrm{TH} 2}=0.46 \mathrm{keV}$ with $R \approx 6 \mathrm{~km}$ for the emitting area. In this model fit, a slightly harder PL component with the best-fit $\Gamma=1.84$ is obtained. Although the statistical improvement in the spectral fit with the $2 \mathrm{BB}+\mathrm{PL}$ model is marginal (the F-probability $\sim 0.02$ ), the consideration of a cooler emission component from the neutron star with the canonical size may be useful to understand the physical nature of $0526-66$, and thus we include the results from this $2 \mathrm{BB}+\mathrm{PL}$ model fit in Table 1 .

\section{NUSTAR TIMING ANALYSIS}

Due to poor photon statistics and the large background ( $\sim 500$ counts per FPM in the $2-40 \mathrm{keV}$ band with $\sim 60 \%$ background [including the contamination from N49]), the pulsation search (for the presumed small $f_{p} \lesssim 10 \%$ ) is difficult with our NuSTAR data. Based on the previously reported values of $\dot{P}$ and $P$, we calculated $Z_{1}^{2}$ statistic as a function of frequency within a plausible range of $P=8.044-8.076 \mathrm{~s}$ (Güver et al. 2012) using events from both FPMA and FPMB with the arrival times corrected for the Earth and spacecraft motion. We did not find any statistically significant signal with the two largest values of $Z_{1, \max }^{2} \approx 10$ (both are found near $8.05348 \mathrm{~s}$ ). Therefore, we calculate an upper limit on the pulsed fraction (for a sinusoidal pulse profile with a single peak per period) as $f_{p, n \sigma}^{\text {obs }} \approx 2\left[\ln \left(\mathcal{N} / P_{n}\right) / N\right]^{1 / 2}$ where, $N=1159$ is the total number of events (source plus background), $\mathcal{N}=183$ is the number of independent frequency trials, and $P_{n}=\operatorname{erfc}(n / \sqrt{2}) \approx(2 / \pi)^{1 / 2} e^{-n^{2} / 2} / n$ (for $n>2$ ) with $n$ being the confidence level in units of standard deviation $\sigma$. For $n=3, P_{n} \approx 0.003$ and, hence, $f_{p, 3 \sigma}^{\text {obs }}=0.195$ is an upper limit on the observed pulsed fraction at $3 \sigma$ confidence. The corresponding intrinsic pulsed fraction is $f_{p, 3 \sigma}^{\text {int }}=f_{p, 3 \sigma}^{\text {obs }}\left(N / N_{\mathrm{bg}}\right)=0.48$ because the background is contributing $60 \%$ of the total events within the source extraction aperture, $r=30^{\prime \prime}$, in the 2 - $40 \mathrm{keV}$ band.

\section{DISCUSSION}

\subsection{Broadband X-Ray Spectral Nature of 0526-66}

Based on our NuSTAR and Chandra data analysis, we find that a PL-like nonthermal spectral component, in addition to the soft thermal BB component, is required to adequately fit the broadband X-ray spectrum of 0526-66. In our BB + PL model fits, a BB temperature of $k T \approx 0.43 \mathrm{keV}$ is estimated, where the implied radius of the emission region $(R \approx 6.5 \mathrm{~km}$ at the distance of $d=50 \mathrm{kpc}$ ) is smaller than the canonical neutron star size $(R \sim 10 \mathrm{~km})$. The best-fit $2 \mathrm{BB}+$ $\mathrm{PL}$ model shows a lower BB temperature of $k T=0.24$ 
$\mathrm{keV}$, assuming the neutron star radius of $R_{\mathrm{NS}}=10 \mathrm{~km}$. The cooler $(k T=0.24 \mathrm{keV})$ component BB flux contributes $\sim 15 \%$ of the total BB luminosity in the $0.5-$ $5 \mathrm{keV}$ band. Thus, for both of the $\mathrm{BB}+\mathrm{PL}$ and $2 \mathrm{BB}$ $+\mathrm{PL}$ model fits, the $\mathrm{BB}$ component with $k T \sim 0.4-$ $0.5 \mathrm{keV}$ dominates the thermal component X-ray emission of 0526-66. Spin-down ages of $\tau_{s d} \sim 2000-3400 \mathrm{yr}$ have been estimated for 0526-66 (Kulkarni et al. 2003; Tiengo et al. 2009; Güver et al. 2012). While the physical association between SGR 0526-66 and SNR N49 is in debate (see Section 5.2), these spin-down ages are in plausible agreement (within a factor of $\sim 2$ ) with the estimated Sedov age of SNR N49 $\left(\tau_{\text {Sedov }} \sim 4800 \mathrm{yr}\right.$, Park et al. 2012). Thus, we may estimate the age of 0526 66 to be $\tau \sim 10^{3-4} \mathrm{yr}$. If either of these BB temperatures corresponds to that of the passively cooling neutron star's surface after its birth, 0526-66 is hotter than the predictions of the cooling curves for neutron stars of this age (Yakovlev \& Pethick 2004). For a neutron star in this age range, the estimated high surface temperature may suggest a re-heating of the surface, probably due to the recent burst activity, and/or strong magnetic field effects on the thermal evolution of the neutron star (Pons et al. 2009; Viganó et al. 2013).

Based on our best-fit spectral models, we estimate that the hardness ratio for the unabsorbed X-ray flux ( $\mathrm{HR}=f_{15-60 \mathrm{keV}} / f_{1-10 \mathrm{keV}}$ as utilized by Enoto et al. [2017]) is $\mathrm{HR} \sim 0.3-0.4$ (depending on the adopted model). The latest estimates for the time-derivative of the pulsation period, the magnetic field strength, and the characteristic age for $0526-66$ are $\dot{P} \sim 4 \times 10^{-11} \mathrm{~s}$ $\mathrm{s}^{-1}, B \sim 4 \times 10^{14} \mathrm{G}$, and $\tau_{s d} \sim 3200 \mathrm{yr}$, respectively (Güver et al. 2012). We compare our estimated HR values for 0526-66 with the PL-like empirical relations with $\dot{P}, B$, and the neutron star's age, which were suggested based on the Galactic sample of a dozen magnetars (Enoto et al. 2017). Our estimated range of HR for 0526-66 is lower than HR $\sim 1$ that is predicted by Enoto et al. (2017). $\dot{P}$ (and thus $B$ ) for $0526-66$ appears to be decreasing between 2000 and 2009 (Kulkarni et al. 2003; Tiengo et al. 2009; Güver et al. 2012). The soft X-ray flux of 0526-66 has been decaying between 1992 and 2009 (Park et al. 2012; Güver et al. 2012). It is difficult to estimate changes in the BB temperature of $0526-66$ between 2009 and 2018. The NuSTAR's detector response is limited to $E>2 \mathrm{keV}$, and the soft X-ray spectrum (at $E \lesssim 5 \mathrm{keV}$ ) of $0526-66$ in the $N u S T A R$ data is significantly contaminated by that from SNR N49. Based on the $\mathrm{BB}+\mathrm{PL}$ model fit to our NuSTAR data alone with an assumption that the BB emitting area stays the same as it was in 2009, we place a $2 \sigma$ upper limit of $k T_{B B} \lesssim 0.5 \mathrm{keV}$ for the thermal component emission of 0526-66 as of 2018. Considering the possible temporal evolution in $\dot{P}, B$, and $k T$, we speculate that it might be partially responsible for the low HR. Our estimated limits on $k T_{B B}$ and $f_{p}$ as of 2018 based on our $N u S T A R$ data are not constraining due to poor photon statistics. Significantly deeper $N u S T A R$ and Chandra observations would be required to constrain the recent evolution of these critical neutron star parameters.

Our spectral fits indicate the photon index $\Gamma \sim 2$ for the PL component which dominates the hard X-ray spectrum of $0526-66$ at $E \gtrsim 10 \mathrm{keV}$. This spectral slope of the
$\mathrm{PL}$ component is larger than those measured for other Galactic magnetars $(\Gamma \lesssim 1.5$, e.g., Enoto et al. [2017]). Apparently, 0526-66 has not shown any activity since its giant bursts in 1979, being in the quiescent state (probably with a steady cooling) for the longest time period among the observed magnetars. We speculate that this distinctive evolutionary state of 0526-66 (compared with that for other magnetars with more frequent burst activity) in the last several decades might have led to a steeper nonthermal spectral component, and thus somewhat lower HR in the latest broadband X-ray spectrum. The best-fit PL photon index for $0526-66$ is similar to that of the synchrotron emission spectrum from the relativistically accelerated electrons in pulsar magnetospheres, or from pulsar wind nebulae (PWNe, although some PWNe show harder spectra with $\Gamma \sim 1.0-1.5)$. However, the high-resolution Chandra ACIS images show no evidence for an extended nebulosity around the pointlike source 0526-66 (Kulkarni et al. 2003; Park et al. 2012). If we entertained the intriguing possibility of a PWN being developed for 0526-66, the lack of an observed extended nebula in the Chandra data would require an angular size of $\lesssim 1^{\prime \prime}$ that cannot be resolved by Chandra. The corresponding physical size is $\lesssim 0.2 \mathrm{pc}$ at the distance of the LMC $(d=50 \mathrm{kpc})$, which would be similar to (or smaller than) those of the torus and jet associated with young pulsars found in the Galactic SNRs G292.0+1.8 (Park et al. 2007) and 3C58 (Slane et al. 2002).

\subsection{Notes on Foreground Absorption for 0526-66}

SGR 0526-66 is projected within the boundary of SNR N49 in the LMC (Cline et al. 1982; Rothschild et al. 1994). However, the physical association between the two has not been conclusive. The statistical chance probability for a coincidental alignment along the line of sight between N49 and 0526-66 is not negligible (Gaensler et al. 2001). If the massive progenitor of 0526-66 was born in a nearby massive stellar cluster, a significantly older age $\left(\sim 10^{5}\right.$ yr $)$ than that of N49 ( $\sim 4800 \mathrm{yr})$ is implied for 0526-66 (Klose et al. 2004). In our spectral model fits, the contribution from the $\mathrm{PL}$ component is considerable in the soft band X-ray flux (at $E<2 \mathrm{keV})$ as well as in the hard band $(E>5 \mathrm{keV})$. This results in an inferred foreground LMC column $N_{\mathrm{H}, \mathrm{LMC}}$ $\sim 3 \times 10^{21} \mathrm{~cm}^{-2}$ toward 0526-66 (Table 1), a factor of $\sim 2$ larger than that estimated for SNR N49. This higher LMC column for 0526-66 might cast further doubt on its physical association with N49. When we fixed the LMC column at the value estimated for N49 $\left(N_{\mathrm{H}, \mathrm{LMC}} \sim\right.$ $1.5 \times 10^{21} \mathrm{~cm}^{-2}$, Park et al. 2012), the overall spectral model fits (for those models presented in Table 1) became slightly poorer $\left(\chi^{2} / \nu \sim 1.14\right)$. Although these fits may be formally distinguished from those summarized in Table 1 (e.g., $F$-probability $\sim 6$ and $1 \times 10^{-4}$, for the $\mathrm{BB}+\mathrm{PL}$ and $2 \mathrm{BB}+\mathrm{PL}$ model fits, respectively), the overall fits may still be considered to be statistically acceptable. Also, due to the model-dependence of these $N_{\mathrm{H}, \text { LMC }}$ estimates (between 0526-66 and N49) and the relatively large uncertainties, it may not be straightforward to firmly conclude that the X-ray spectrum of 0526 66 is more absorbed than that of N49.

\section{SUMMARY}


We have confidently detected SGR $0526-66$ in the quiescent state at $E>10 \mathrm{keV}$ with $N u S T A R$. The joint spectral fits to the NuSTAR + Chandra spectrum require at least two component model if BB and PL are used for the individual components. The hard X-ray emission at $E>$ $10 \mathrm{keV}$, which is fitted with a PL spectrum, is most likely nonthermal in origin. The slope of the PL component ( $\Gamma$ $\sim 1.8-2.1$ ) is softer than those for other magnetars observed with NuSTAR. For our best-fit BB + PL model, we obtain a high $\mathrm{BB}$ temperature of $k T \approx 0.43 \mathrm{keV}$, and the estimated radius of the $\mathrm{X}$-ray-emitting area $(R \approx 6.5$ $\mathrm{km})$ is smaller than the canonical size of a neutron star. If we assumed the BB spectrum from the cooling neutron star with a standard radius of $R_{\mathrm{NS}}=10 \mathrm{~km}$ in addition to the hot $k T \sim 0.4 \mathrm{keV}$ component, a lower BB temperature of $k T \approx 0.24 \mathrm{keV}$ is obtained for the passively cooling surface of the neutron star. The presence of the relatively soft PL component in the X-ray spectrum of 0526-66 may provide an intriguing opportunity to study the magneto-thermal evolution of a magnetar during the substantially long quiescent period of several decades after the strong outbursts. Based on the NuSTAR data, we place a $3 \sigma$ upper limit of $f_{p} \sim 0.48$ on the intrinsic pulsed fraction of $0526-66$ in the $2-40 \mathrm{keV}$ band.

We thank the anonymous referee for her/his comments that helped improving this manuscript. This work has been supported in part by NASA NuSTAR grant 80NSSC17K0633 and the Chandra grant GO9-0072A to the University of Texas at Arlington. O. K. was supported in part by NASA through Chandra Award number TM8-19005B issued by the Chandra X-Ray Center which is operated by the Smithsonian Astrophysical Observatory for and on behalf of NASA under the contract NAS8-03060.

\section{REFERENCES}

Anders, E., \& Grevesse, N. 1989, Geochimica et Cosmochimica Acta, 53, 197

Cline, T. L., Desai, U. D., Pizzichini, G., Teegarden, B. J., Evans, W. D., Klebesadel, R. W., Laros, J. G., Hurley, K., Niel, M., \& Vedrenne, G. 1980, ApJ, 237, L1

Cline, T. L., Desai, U. D., Teegarden, B. J., Evans, W. D., Klebesadel, R. W., Laros, J. G., Barat, C., Hurley, K., et al. 1982, ApJ, 255, L45

Dickey, J. M., \& Lockman, F. J. 1990, ARA\&A, 28, 215

Enoto, T. et al. 2017, ApJS, 231, 8

Gaensler, B. M., Slane, P. O., Gotthelf, E. V., \& Vasisht, G. 2001, ApJ, 559, 963

Gotthelf, E. V. et al. 2019, ApJ, 874, 25

Güver, T., Göğüs, E., \& Özel, F. 2012, MNRAS, 424, 210

Ho, W. C. G., Potekhin, A. Y., \& Chabrier, G., 2008, ApJS, 178, 102

Hughes, J. P., Hayashi, I., \& Koyama, K. 1998, ApJ, 505, 732

Klose, S. et al. 2004, ApJ, 609, L13

Kulkarni, S. R., Kaplan, D. L., Marshall, H. L., Frail, D. A., Murakami, T., \& Yonetoku, D. 2003, ApJ, 585, 948

Maggi, P., Haberl, F., Kavanagh, P. J., Sasaki, M., Bozzetto, L. M., Filipović, M. D., Vasilopoulos, G., Pietsch, W., Points, S. D., Chu, Y. -H., Dickel, J., Ehle, M., Williams, R., \& Greiner, J. 2016, A\&A, 585A, 162
Mazets, E. P., Golentskii, S. V., Ilinskii, V. N., Aptekar, R. L., \& Guryan, Iu. A. 1979, Nature, 282, 587

Mori, K. \& No, W. C. G, 2007, MNRAS, 377, 905

Park, S., Burrows, D. N., Garmire, G. P., Nousek, J. A., Hughes, J. P., \& Williams, R. M. 2003, ApJ, 586, 210

Park, S., Hughes, J. P., Slane, P. O., Burrows, D. N., Gaensler, B. M., \& Ghavamian, P. 2007, ApJ, 670, L121

Park, S., Hughes, J. P., Slane, P. O., Burrows, D. N., Lee, J.-J., \& Mori, K. 2012, ApJ, 748, 117

Pavlov, G.G., Shibanov, Y. A. Zvlin, V. E., \& Meyer, R. D. 1995,

in "The Lives of the Neutron Stars," ed. M.A. Alpar, U.

Kiziloglu, \& J. van Paradijs (NATO ASI Ser. C, 450; Dordrecht: Kluwer), p. 71

Pons, J. A., Miralles, J. A., \& Geppert, U. 2009, A\&A, 496, 207

Rothschild, R. E., Kulkarni, S. R., \& Lingenfelter, R.E. 1994,

Nature, 368, 432

Russell, S. C., \& Dopita, M. A., 1992, ApJ, 384, 508

Schenck, A., Park, S., \& Post, S. 2016, AJ, 151, 161

Slane, P. O., Helfand, D. J., \& Murray, S. S. 2002, ApJ, 571, L45

Tiengo, A., Esposito, P., Mereghetti, S., Israel, G. L., Stella, L., Turolla, R., Zane, S., Rea, N., Götz, D., \& Feroci, M. 2009, MNRAS, 399L, L74

Uchida, H., Koyama, K., \& Yamaguchi, H. 2015, ApJ, 808, 77

Viganó, D., Rea, N., Pons, J. A., Perna, R., Aguilera, D. N., \& Miralles, J. A., 2013, MNRAS, 434, 123

Yakovlev, D. G., \& Pethick, C. J., 2004, ARA\&A, 42, 169 
TABLE 1

SUMMARY OF SPECTRAL MODEL FITS TO SGR 0526-66

\begin{tabular}{lcc}
\hline \hline \multicolumn{1}{c}{ Model Parameter } & $\mathrm{BB}+\mathrm{PL}$ & $2 \mathrm{BB}+\mathrm{PL}$ \\
\hline$k T_{\mathrm{TH} 1}(\mathrm{keV})$ & $0.43 \pm 0.01$ & $0.24_{-0.06}^{+0.05}$ \\
$k T_{\mathrm{TH} 2}(\mathrm{keV})$ & - & $0.46_{-0.03}^{+0.05}$ \\
$\Gamma$ & $2.10_{-0.22}^{+0.16}$ & $1.84 \pm 0.24$ \\
$R_{\mathrm{TH} 1}(\mathrm{~km})$ & $6.5 \pm 0.5$ & $10($ fixed $)$ \\
$R_{\mathrm{TH} 2}(\mathrm{~km})$ & - & $5.9_{-1.3}^{+0.8}$ \\
$f_{\mathrm{TH}}{ }^{\mathrm{a}}\left(0.5-5 \mathrm{keV}, 10^{-13} \mathrm{erg} \mathrm{cm}^{-2} \mathrm{~s}^{-1}\right)$ & $4.5_{-0.8}^{+0.5}$ & $5.7_{-1.2}^{+0.7}$ \\
$f_{\mathrm{PL}}{ }^{\mathrm{a}}\left(5-40 \mathrm{keV}, 10^{-13} \mathrm{erg} \mathrm{cm}^{-2} \mathrm{~s}^{-1}\right)$ & $4.4_{-1.4}^{+0.5}$ & $5.3_{-4.6}^{+0.8}$ \\
$L_{\mathrm{TH}}{ }^{\mathrm{b}}\left(0.5-5 \mathrm{keV}, 10^{35} \mathrm{erg} \mathrm{s}^{-1}\right)$ & $1.8_{-0.3}^{+0.2}$ & $2.2_{-0.5}^{+0.3}$ \\
$L_{\mathrm{PL}}{ }^{\mathrm{O}}\left(5-40 \mathrm{keV}, 10^{35} \mathrm{erg} \mathrm{s}^{-1}\right)$ & $1.3_{-0.4}^{+0.1}$ & $1.6_{-1.4}^{+0.2}$ \\
$f_{\mathrm{X}}^{\mathrm{a}}\left(0.5-60 \mathrm{keV}, 10^{-13} \mathrm{erg} \mathrm{cm}^{-2} \mathrm{~s}^{-1}\right)$ & $14.1_{-4.2}^{+2.1}$ & $15.4_{-5.9}^{+1.7}$ \\
$L_{\mathrm{X}}{ }^{\mathrm{b}}\left(0.5-60 \mathrm{keV}, 10^{35} \mathrm{erg} \mathrm{s}^{-1}\right)$ & $5.2_{-1.6}^{+1.2}$ & $5.4_{-3.4}^{+1.0}$ \\
$N_{\mathrm{H}, \mathrm{LMC}}\left(10^{21} \mathrm{~cm}^{-2}\right)$ & $3.4 \pm 1.0$ & $2.7_{-0.6}^{+0.7}$ \\
$\chi^{2} / \nu$ & $305.5 / 279$ & $299.8 / 278$ \\
\hline
\end{tabular}

Note. - Uncertainties are with a $90 \%$ C.L. The Galactic column is fixed at $N_{\mathrm{H}, \mathrm{Gal}}=6 \times 10^{20} \mathrm{~cm}^{-2} . d=50 \mathrm{kpc}$ is assumed.

a The observed flux.

b The X-ray luminosity after removing the absorption.

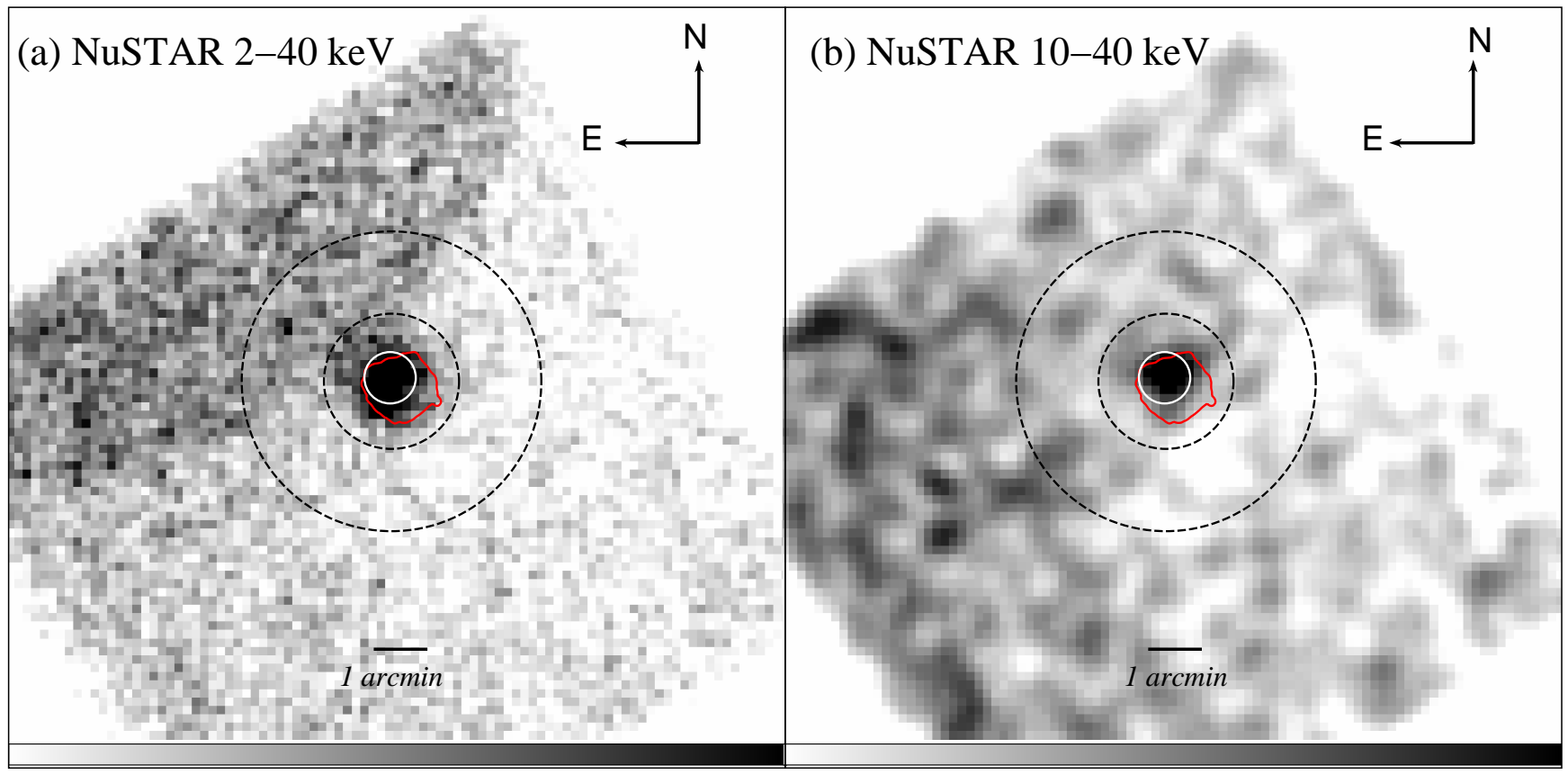

Fig. 1.- (a) Grey-scale NuSTAR images of SGR 0526-66: (a) the $2-40 \mathrm{keV}$ band, and (b) the $10-40 \mathrm{keV}$ band. For the purposes of display both images have been binned by $4 \times 4$ pixels. In (b), the image has also been smoothed. In (a) and (b), our source region for 0526-66 is marked with a white circle. The annular background region is marked with dashed circles. The outer boundary of the LMC SNR N49 (taken from the archival Chandra ACIS data) is shown with red contours. 

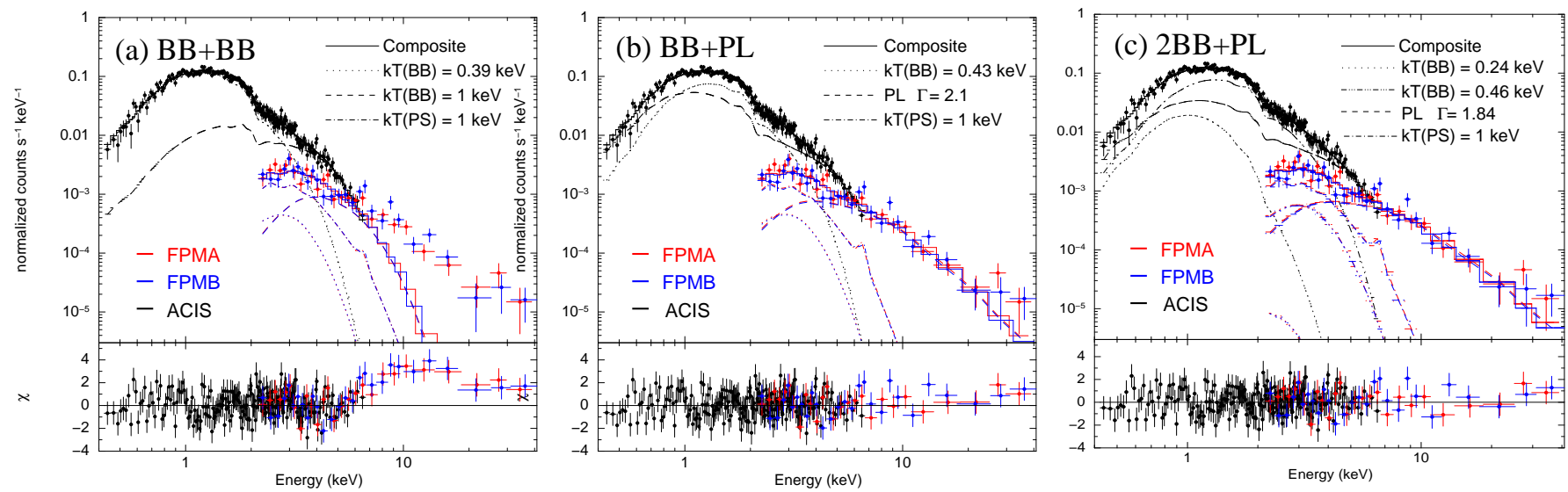

Fig. 2.- The observed NuSTAR and Chandra spectra of 0526-66. The best-fit models are overlaid in each panel: (a) 2BB, (b) BB+PL, and (c) $2 \mathrm{BB}+\mathrm{PL}$. The PS component (with $k T=1 \mathrm{keV}$, representing the contamination by thermal X-ray emission from SNR N49) is shown for the NuSTAR spectrum. The lower panels are residuals from the best-fit model.
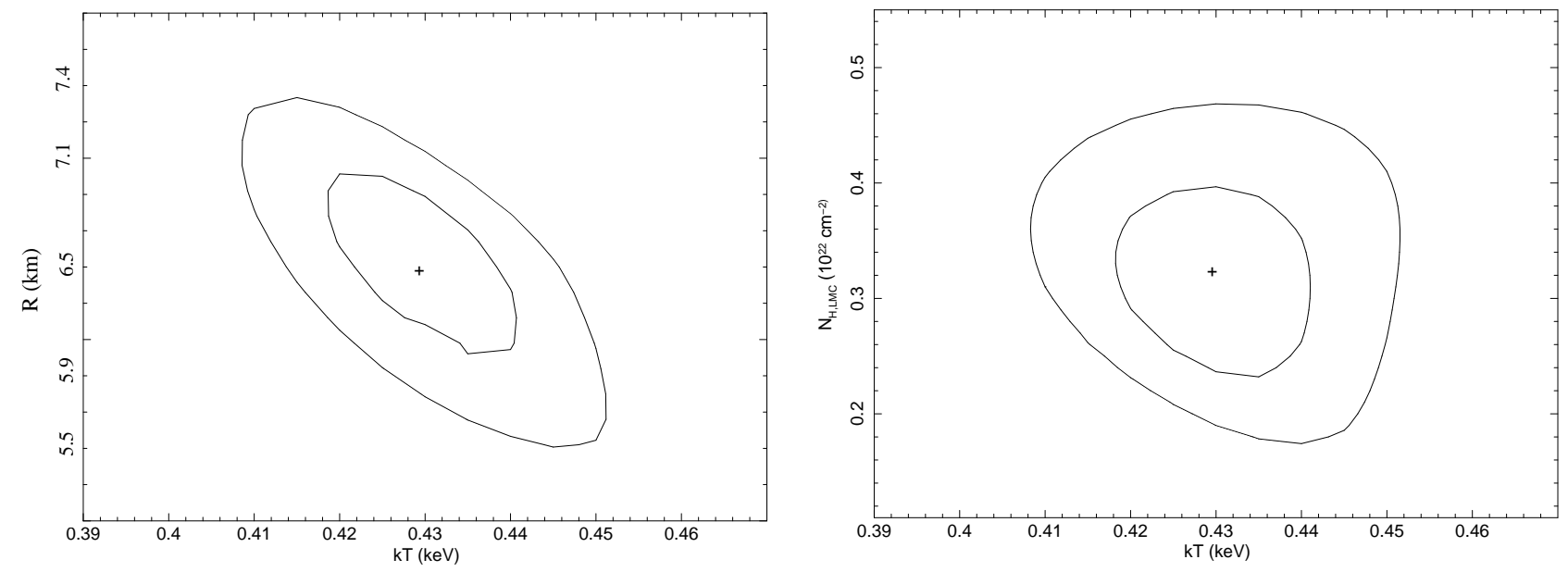

Fig. 3.- (a) The BB temperature vs. emission region radius (at $d=50 \mathrm{kpc}$ ), and (b) the BB temperature vs. $N_{\mathrm{H}, \mathrm{LMC}}$ contour plots based on our best-fit BB + PL model (Table 1). In (a) and (b), $90 \%$ and $99 \%$ contours are shown. The best-fit temperature and emitting radius are marked with a cross. 